\title{
Emergency medical services key performance measurement in Asian cities
}

\author{
Nik Hisamuddin Rahman ${ }^{1 *}$, Hideharu Tanaka², Sang Do Shin ${ }^{3}$, Yih Yng Ng${ }^{4}$, Thammapad Piyasuwankul ${ }^{5}$, \\ Chih-Hao Lin ${ }^{6}$ and Marcus Eng Hock Ong ${ }^{7}$
}

\begin{abstract}
Background: One of the key principles in the recommended standards is that emergency medical service (EMS) providers should continuously monitor the quality and safety of their services. This requires service providers to implement performance monitoring using appropriate and relevant measures including key performance indicators. In Asia, EMS systems are at different developmental phases and maturity. This will create difficultly in benchmarking or assessing the quality of EMS performance across the region. An attempt was made to compare the EMS performance index based on the structure, process, and outcome analysis.
\end{abstract}

Findings: The data was collected from the Pan-Asian Resuscitation Outcome Study (PAROS) data among few Asian cities, namely, Tokyo, Osaka, Singapore, Bangkok, Kuala Lumpur, Taipei, and Seoul. The parameters of inclusions were broadly divided into structure, process, and outcome measurements. The data was collected by the site investigators from each city and keyed into the electronic web-based data form which is secured strictly by username and passwords. Generally, there seems to be a more uniformity for EMS performance parameters among the more developed EMS systems. The major problem with the EMS agencies in the cities of developing countries like Bangkok and Kuala Lumpur is inadequate or unavailable data pertaining to EMS performance.

Conclusions: There is non-uniformity in the EMS performance measurement across the Asian cities. This creates difficulty for EMS performance index comparison and benchmarking. Hopefully, in the future, collaborative efforts such as the PAROS networking group will further enhance the standardization in EMS performance reporting across the region.

Keywords: EMS; Pre-hospital care; Performance; Benchmarking

\section{Findings \\ Background}

In today's health care environment, the demand for objective comparative information about the performance of health care organizations and providers has created a need for data-driven evaluation processes [1]. As a part of the health care environment, pre-hospital emergency medical services (EMS) systems are no different in their need for objective comparative system information to assist government officials at all levels to establish relevant policy, select appropriate system design, and monitor system quality and effectiveness. Assessing EMS performance may be a simple task if it is carried out within

\footnotetext{
* Correspondence: nhliza@hotmail.com

${ }^{1}$ Department of Emergency Medicine, School of Medical Sciences, University Sains Malaysia, Kota Bharu 16150, Malaysia

Full list of author information is available at the end of the article
}

a local health care system, but things can get complicated if the comparison is made between states or countries $[2,3]$. Different states or countries may deliver EMS differently. In Asia, EMS systems are at different developmental phases and maturity. For example, Singapore, Japan, and Korea have mature and systematic EMS whereas in other developing countries, the system is still at its infancy $[4,5]$. This will create difficultly benchmarking or assessing the quality of EMS performance across the region. Despite limited data, we attempted to compare basic EMS performance based on reports by the Pan-Asian Resuscitation Outcome Study (PAROS) group [6]. Hopefully, this will be the first step in understanding the quality of EMS in Asia and the stepping stone for benchmarking in the future.

\section{Springer}

(C) 2015 Rahman et al.; licensee Springer. This is an Open Access article distributed under the terms of the Creative Commons Attribution License (http://creativecommons.org/licenses/by/4.0), which permits unrestricted use, distribution, and reproduction in any medium, provided the original work is properly credited. 


\section{EMS performance measurement}

Measuring quality in EMS systems is challenging [7]. Measuring quality in EMS is important since EMS is the practice of medicine in the pre-hospital setting. The goal of EMS Performance Measurement (EMSPM) is to apply knowledge, data, and experience to evaluate and improve EMS service delivery, personnel performance, and clinical care. The need for increased coordination in patient care and higher quality care at lower costs has made it essential for EMS agencies to have in-place quality control or quality improvement programs that rely on key performance indicators to continuously monitor the system's overall performance and the effectiveness of the different pre-hospital interventions $[8,9]$. The Institute of Medicine (IOM), in a report entitled "Emergency Medical Services at the Crossroads" and published in 2006, recommended the development of "evidence based performance indicators that can be nationally standardized so that statewide and national comparisons can be made" [10]. The development and implementation of these indicators would enhance accountability in EMS and provide EMS agencies with data to measure their system's overall performance and to develop sound strategic quality improvement planning.

\section{Performance indicators}

Performance indicators are measurement tools that should be "specific, measurable, action oriented, relevant and timely" [11]. An indicator is a metric that reflects on the performance of a system or process. As the indicator value rises or falls, it suggests that the system or process is operating better or worse - like a performance thermometer. The common measurable indicators include structure, process, and outcome. (Table 1) However, the National Highway and Traffic Safety Act (NHTSA) has recommended a more comprehensive measurement which includes system design and structure, human resources (culture, training, safety, credentialing, etc.), clinical care and outcome, response, finance, quality management, and community demographics [12]. Problems may arise from a complex measurement system especially when the system is used for multiple agencies or international usage. Different agencies or countries may have different definitions for a specific measure such as response time, or the data may not be easily available within that particular community (such as pre-hospital defibrillation) $[13,14]$. This will create standardization of EMSPM across the region really difficult. Finding the most common available denominators is perhaps the best method to carry out the inter-agency EMSPM [15].

The structure of EMS agencies differs significantly between the Asian countries. The focus of emergency health care in most of the Asian countries relies heavily on the hospital system, with little emphasis on pre-hospital care other than rapid transport. The quality of care available at all levels appears to be directly related to the economic status of that country and the efforts of government and health authorities to identify and support the development of pre-hospital services. Countries like Singapore, South Korea, Japan, and Taiwan have a much more mature EMS setup compared to other countries in the Asian region $[16,17]$. They have robust data system collection partly for the purpose of quality improvement. Unfortunately, there are no standardized quality assurance and monitoring system within this region. Overall, comparing the EMSPM is difficult partly due to lack of data and nonstandardization of the delivery system. However, we attempted to collect basic common data within the PAROS study group in 2013.

\section{Methods}

The data collected comprises of structure, process, and outcome information of the EMS system within each of the EMS agencies. A survey form was created and sent via email to all the representatives of the EMS agencies or the medical directors of the PAROS group. Some of the survey forms were handed to the participating members by hand during the annual PAROS meetings held in various cities within Asia. Some of the data was obtained from the previous publications within the PAROS group that is adopted from cardiac arrest registry to enhance survival (CARES) study based in the USA [18,19]. Twenty-four variables were assessed and compared, all of which were derived by consensus across the PAROS countries. The components were agreed upon after series of meeting and followed the Utstein template. The most common available denominators of measurement were taken into analysis and comparison. The other variables that were not available in all the participated countries would be excluded from analysis. The overall comparison of EMSPM is carried out based on the countries and cities where the EMS agencies originate from.

\section{Results}

The final response reported in this study is from seven cities (Seoul, Tokyo, Osaka, Taipei, Singapore, Bangkok, and Kuala Lumpur) and six countries (Singapore, South Korea, Taiwan, Japan, Thailand, and Malaysia). General demography of each of the cities is as shown in Table 2. In all, we managed to gather measurement indicators for 13 structural components, nine process components, and two outcome indicators. Korea, Singapore, Taiwan, and Japan provided the data from their existing EMS registries whereas countries like Malaysia and Thailand based their data mainly from hospitals within the big cities like Kuala Lumpur and Bangkok. Some of the data is national and others are specific only for the cities (Tables 3, 4, and 5). 
Table 1 Performance indicators

\begin{tabular}{|c|c|c|}
\hline Indicator type & Definitions & EMS systems performance index examples \\
\hline \multirow[t]{7}{*}{ Structure } & \multirow[t]{7}{*}{ Characteristics of the different components of the system } & (i) Facilities \\
\hline & & (ii) Equipment \\
\hline & & (iii) Staffing \\
\hline & & (iv) Knowledge base of providers \\
\hline & & (v) Credentials \\
\hline & & (vi) Deployment \\
\hline & & (vii) Response times \\
\hline \multirow[t]{3}{*}{ Process } & \multirow{3}{*}{$\begin{array}{l}\text { Combination or sequence of steps in patient care intended to } \\
\text { improve patient outcome }\end{array}$} & (i) Medical protocols \\
\hline & & (ii) Medication administration \\
\hline & & (iii) Transport to appropriate facility \\
\hline \multirow[t]{7}{*}{ Outcome } & Changes in health and well-being related to antecedent care $6 \mathrm{Ds}^{\mathrm{a}}$ & \\
\hline & (i) Death & (i) Out of hospital cardiac arrest survival \\
\hline & (ii) Disease & (ii) Patient Satisfaction \\
\hline & (iii) Disability & (iii) Improvement in pain score \\
\hline & (iv) Discomfort & \\
\hline & (v) Dissatisfaction & \\
\hline & (vi) Destitution & \\
\hline
\end{tabular}

${ }^{\mathrm{a}}$ EMS outcomes defined by Emergency Medical Services Outcomes Project (EMSOP).

\section{Discussion}

Generally, there seems to be a more uniformity for EMS performance parameters among the more developed EMS systems. The major problem with the EMS agencies in the cities of developing countries like Bangkok and Kuala Lumpur is inadequate or unavailable of data pertaining to EMS performance. This is partly due to lack of uniform and standardized data collection. EMS agencies in Seoul, Tokyo, Osaka, Taipei, and Singapore utilize established electronic patient care record systems for all EMS cases [20]. This allows a more consistent and sustainable data collection for multiple purposes, including for performance analysis. Unfortunately, the EMS agencies in Bangkok and Kuala Lumpur do not use similar electronic patient care record systems. Most patient care records are manual, and data collection is not standardized and non-uniform. Interestingly, the obvious difference in the structure of EMS between the developed and developing countries in Asia lies with the EMS providers and their location. The cities in the more developed countries utilize the fire department and their personnel as the providers whereas the cities in the developing countries prefer hospital-based EMS provision. Similarly, the training hours for the EMS providers for each city differ significantly, partly due to different training legislation and requirements. The ratio of the number of ambulance to the number of providers varies from 2.16 in Bangkok to 0.05 in Taipei. Such data is unavailable for Kuala Lumpur. Mean response time for out-of-hospital cardiac arrest (OHCA) is within $10 \mathrm{~min}$ for all the cities in the developed countries. The response time is much longer in Kuala Lumpur and unavailable for Bangkok. Singapore reported the highest pre-hospital defibrillation rate compared to other cities but unfortunately, the survival to discharge is fairly low. No such data is available for Bangkok and Kuala Lumpur.

There are many challenges in reporting the core measures of EMS performance. Variability in data storage and core measure definitions is very common. Not all countries use a systematic database registry system, especially in the developing countries. Not all data systems utilized similar data dictionaries. This resulted in disparity in the way measurements were derived. Some EMS agencies followed the measure exactly, while

Table 2 General demography of the cities in the survey

\begin{tabular}{llllllll}
\hline City & Seoul & Tokyo & Osaka & Taipei & Singapore & Bangkok & Kuala Lumpur \\
\hline Population & $10,140,000$ & $13,185,502$ & $2,666,371$ & $2,652,959$ & $5,399,200$ & $8,280,925$ & $1,627,172$ \\
Area $\left(\mathrm{km}^{2}\right)$ & 605.21 & $2,187.66$ & 223.00 & 271.799 & 716.1 & $1,568.737$ & 243 \\
Population density per km & 2 & 17,000 & 6,000 & 11,759 & 9,586 & 7,540 & 5,300 \\
Number of districts & 25 & 23 wards & 24 wards & 12 & 28 & 50 & 11 \\
\hline
\end{tabular}


Table 3 Structure comparisons of EMS systems in Asia

\begin{tabular}{|c|c|c|c|c|c|c|c|}
\hline City & Seoul & Tokyo & Osaka & Taipei & Singapore & Bangkok & Kuala Lumpur \\
\hline Operation of ambulance & Fire department & Fire department & Fire department & Fire department & Fire department & Hospital & Hospital plus NGOs \\
\hline Dispatch center & Fire department & Fire department & Fire department & Fire department & Fire department & Fire department & Hospital and NGOs \\
\hline Dispatcher certification & Certified & Certified & Certified & Certified & Certified & Certified & Certified \\
\hline Tiered response & BLS & BLS & BLS & BLS plus ALS & BLS & BLS plus ALS & BLS or ALS \\
\hline Medical direction & Direct only & Mixed & Mixed & Mixed & Indirect only & Direct only & Direct only \\
\hline Dispatcher type & Firefighters & Firefighters & Firefighters & Firefighters & Firefighters & Nurse & EMD \\
\hline Ambulance personnel & EMT & First aider, EMT & First aider, EMT & EMT paramedic & EMT intermediate & Nurse, EMT & Medical assistant, nurses \\
\hline Ambulance station & Fire department & Fire department & Fire department & Fire department & Fire station & Hospital/clinic & Hospital/clinic \\
\hline Total EMS providers & 751 & 2,184 & 600 & 1,452 & 200 & 12 & NA \\
\hline Training period & $>320 \mathrm{~h}$ for EMT-basic & 750 to $1,095 \mathrm{~h}$ for EMT & 750 to $1,095 \mathrm{~h}$ for EMT & 1,280 h for EMT-P & 2,640 h for EMT-I & 6 months & $120 \mathrm{~h}$ \\
\hline Ambulance total number & 117 & 229 & 72 & 76 & 40 & 26 & 60 \\
\hline Ambulance stations total numbers & 114 & 229 & 50 & 43 & 31 & 2 & 6 \\
\hline
\end{tabular}


Table 4 Process comparison of EMS systems in Asia

\begin{tabular}{|c|c|c|c|c|c|c|c|}
\hline City & Seoul & Tokyo & Osaka & Taipei & Singapore & Bangkok & Kuala Lumpur \\
\hline The use of AED & Yes & Yes & Yes & Yes & Yes & Yes & Yes \\
\hline $\begin{array}{l}\text { Mean call to arrival at scene } \\
\text { for OHCA (min/sd) }\end{array}$ & $6.8( \pm 3.5)$ & $6.0( \pm 4.2)$ & $7.8( \pm 3.6)$ & $7.0( \pm 10.0)$ & $10.2( \pm 4.3)$ & $11.8( \pm 6.0)$ & $22.5( \pm 16.0)$ \\
\hline $\begin{array}{l}\text { Mean call to arrival to hospital } \\
\text { for OHCA (min/sd) }\end{array}$ & $21.4( \pm 8.8)$ & $29.0( \pm 9.9)$ & $23.4( \pm 14.1)$ & $15.0( \pm 21.0)$ & $35.5( \pm 9.5)$ & $41.8( \pm 21.0)$ & $42.9( \pm 32.5)$ \\
\hline Dispatcher CPR instruction & Yes & Yes & Yes & Yes & Yes & Yes & Yes \\
\hline Medical oversight of dispatch & Yes & Yes & Yes & Yes & Yes & Yes & Yes \\
\hline Airway management & $\begin{array}{l}\text { Basic and } \\
\text { advance }\end{array}$ & $\begin{array}{l}\text { Basic and } \\
\text { advance }\end{array}$ & $\begin{array}{l}\text { Basic and } \\
\text { advance }\end{array}$ & $\begin{array}{l}\text { Basic and } \\
\text { advance }\end{array}$ & $\begin{array}{l}\text { Basic and } \\
\text { LMA }\end{array}$ & Basic & $\begin{array}{l}\text { Basic and } \\
\text { advance }\end{array}$ \\
\hline EMS-treated OHCA (\%) & $100 \%$ & $100 \%$ & $100 \%$ & $100 \%$ & $100 \%$ & NA & NA \\
\hline Pre hospital defibrillation (\%) & $5.4 \%$ & $8.9 \%$ & $14.3 \%$ & $8.7 \%$ & $21.9 \%$ & NA & NA \\
\hline CPR by EMS (\%) & $90.6 \%$ & $100 \%$ & $100 \%$ & $90.8 \%$ & $100 \%$ & NA & NA \\
\hline
\end{tabular}

NA, no data available.

others had interpreted the measure more loosely. Many local EMS systems use paper pre-hospital patient care records while others use the electronic format. Abstracting information from paper forms is difficult, time-consuming, and not necessarily accurate [21]. In contrast, some software systems have a high degree of technological sophistication that forces users to complete forms before closing the record [22]. Moreover, without training in the specific core measures, users may not have understood the criticality of completing each data point. One of the clear challenges identified was the difficulty and inability by EMS agencies to obtain universal hospital outcome data on every ambulance transport. This was evidenced by the low response rate for specific cardiac arrest outcome measures. These measures relied upon the hospital to report survival to emergency department discharge and survival to hospital discharge. Another significant limitation of reporting EMS information is related to the nature of the "tiered" EMS system that is present in cities like Kuala Lumpur. Because there are EMS first responders and separate ambulance transport units that arrive at a later time, often, two records are initiated for each patient. This inability to aggregate first responder data with transport provider data could lead to a conclusion that care was not provided, when in fact, it may have been provided to the patient by a different provider.

\section{Recommendation}

A few efforts should be considered in order to reduce the limitations in obtaining universal and uniform EMS performance measures in the Asian countries. The establishment of the PAROS in the Asian region in 2010 has significantly changed the landscape of networking among EMS experts in the region. The PAROS is a collaborative research group formed by dedicated pre-hospital emergency care (PEC) providers conducting PEC research in the Asia-Pacific region. The initial step involved the data collection pertaining to the OHCA within the Asian region. Before the study was conducted, basic data on the EMS components were obtained from the involved countries and cities, namely, the general structure, process, and outcome of the EMS system. The regional networking has created standardized definitions across the PAROS network by adopting a consensual common taxonomy and data collection methodology. This will allow consideration in an attempt to create uniform EMS measures in the region and to identify common available core measures from each country or cities. The uniform core measures will create a more meaningful EMS practice comparison and benchmarking in the region. This is a very important step to a very long journey in creating a sustainable unique, low-cost, self-funded model of a collaborative research and education network in EMS. No doubt, there will be a lot more hurdles to be faced but with perseverance and commitment this effort will succeed.

Table 5 Outcome comparison of EMS systems in Asia

\begin{tabular}{llllllll}
\hline City & Seoul & Tokyo & Osaka & Taipei & Singapore & Bangkok & Kuala Lumpur \\
\hline EMS ROSC & $23.4 \%$ & $35.1 \%$ & $35 \%$ & $31.1 \%$ & $11.2 \%$ & $9.1 \%$ & NA \\
Survival to admission for OHCA, n (\%) & $20.4 \%$ & $27.3 \%$ & $28.1 \%$ & $5.9 \%$ & $17.0 \%$ & $27.7 \%$ & $8.0 \%$ \\
Survival to discharge for OHCA, n (\%) & $8.9 \%$ & $5.2 \%$ & $7.6 \%$ & $5.9 \%$ & $3.2 \%$ & $6.8 \%$ & $1.3 \%$ \\
CPC & $3.0 \%$ & $2.8 \%$ & $2.8 \%$ & $3.0 \%$ & $1.7 \%$ & $1.7 \%$ & $\mathrm{NA}$ \\
\hline
\end{tabular}

ROSC, return of spontaneous circulation; OHCA, out-of-hospital cardiac arrest; CPC, cerebral performance score 1 or 2; NA, no available data. 


\section{Conclusion}

EMS is an integral part of every community's total health care delivery system. Consistent evaluation of clinical and response performance indicators are crucial components in ensuring that first response services are operating at peak efficiency. To achieve this, continuous quality improvement (QI) practices must become an essential and seamless part of normal EMS routines. Unfortunately at the present time, there is an obvious significant difference in the EMS practice of reporting in the Asian region. This creates difficulty for EMS performance index comparison and benchmarking. Data systems that are robust and agile, with the ability to report clinical indicators and performance measures, are a key tool in quality improvement activities. Hopefully, in the future, collaborative efforts such as the PAROS networking group will further enhance the standardization in EMS performance reporting across the region.

\section{Abbreviations}

EMS: emergency medical services; PAROS: Pan-Asian Resuscitation Outcome Study; EMSPM: Emergency Medical Services Performance Measurements; IOM: Institute of Medicine; NHTSA: National Highway and Traffic Safety Act; OHCA: out-of-hospital cardiac arrest; PEC: pre-hospital emergency care.

\section{Competing interests}

The authors declare that they have no competing interests.

\section{Authors' contributions}

NHR contributed in the conception of the study, data collection, and manuscript preparation. HT and $\mathrm{MEHO}$ contributed in the data collection and review of manuscript. SDS, YYN, TP, and CHL contributed in the data collection. All authors read and approved the final manuscript.

\section{Acknowledgement}

The authors would like to thank to all the investigators within the Pan-Asian Resuscitation Outcome Study (PAROS) and also convey the greatest appreciation to the Asian EMS Council who had given encouragement and morale support to the preparation of this manuscript.

\footnotetext{
Author details

'Department of Emergency Medicine, School of Medical Sciences, University Sains Malaysia, Kota Bharu 16150, Malaysia. ²Department of EMS System, Graduate School, Kokushikan University, Tokyo, Japan. ${ }^{3}$ Department of Emergency Medicine, College of Medicine, Seoul National University, Seoul, Korea. ${ }^{4}$ Medical Department, Singapore Civil Defence Force, Singapore, Singapore. ${ }^{5}$ Department of Emergency Medicine, Prince of Songkla University, Hat Yai, Thailand. 'Department of Emergency Medicine, College of Medicine, National Cheng Kung University Hospital, National Cheng Kung University, Tainan, Taiwan. ${ }^{7}$ Department of Emergency Medicine, Singapore General Hospital, Singapore, Singapore.
}

Received: 3 October 2014 Accepted: 14 April 2015

Published online: 23 April 2015

\section{References}

1. Silber JH, Rosenbaum PR, Ross RN, Ludwig JM, Wang W, Niknam BA, et al. A hospital-specific template for benchmarking its cost and quality. Health Serv Res. 2014;49(5):1475-97.

2. Ma MH, Chiang WC, Ko PC, Huang JC, Lin CH, Wang HC, et al. Outcomes from out-of-hospital cardiac arrest in Metropolitan Taipei: does an advanced life support service make a difference? Resuscitation. 2007;74(3):461-9. Epub 2007 Apr 25

3. Vaillancourt C, Stiell IG. Canadian Cardiovascular Outcomes Research Team. Cardiac arrest care and emergency medical services in Canada. Can J Cardiol. 2004;20(11):1081-90.
4. Ong ME, Cho J, Ma MH, Tanaka H, Nishiuchi T, Al Sakaf O, et al. Comparison of emergency medical services systems in the pan-Asian resuscitation outcomes study countries: report from a literature review and survey. Emerg Med Australas. 2013;25(1):55-63.

5. Nielsen K, Mock C, Joshipura M, Rubiano AM, Zakariah A, Rivara F. Assessment of the status of prehospital care in 13 low- and middle-income countries. Prehosp Emerg Care. 2012;16(3):381-9.

6. Shin SD, Ong ME, Tanaka H, Ma MH, Nishiuchi T, Alsakaf O, et al. Comparison of emergency medical services systems across pan-Asian countries: a Web-based survey. Prehosp Emerg Care. 2012;16(4):477-96.

7. Harrawood D, Easton K. EMS measuring stick. How to develop an EMS clinical performance scorecard. JEMS. 2000;25(7):38-45.

8. MacFarlane C, Benn CA. Evaluation of emergency medical services systems: a classification to assist in determination of indicators. Emerg Med J. 2003;20(2):188-91.

9. Roudsari BS, Nathens AB, Cameron P, Civil I, Gruen RL, Koepsell TD, et al. International comparison of prehospital trauma care systems. Injury. 2007;38(9):993-1000. Epub 2007 Jul 20.

10. Berger E. Crossroads: report calls for EMS standards of care. Ann Emerg Med. 2006;48(2):142-3.

11. Pittet V, Burnand B, Yersin B, Carron PN. Trends of pre-hospital emergency medical services activity over 10 years: a population-based registry analysis. BMC Health Serv Res. 2014;14(1):380. doi:10.1186/1472-6963-14-380.

12. Mears GD, Pratt D, Glickman SW, Brice JH, Glickman LT, Cabañas JG, et al. The North Carolina EMS Data System: a comprehensive integrated emergency medical services quality improvement program. Prehosp Emerg Care. 2010;14(1):85-94.

13. Cady G. 200 city survey. JEMS 2001 annual report on EMS operational and clinical trends in large, urban areas. JEMS. 2002;27(2):46-65. 68-70.

14. Sayre MR, White LJ, Brown LH, McHenry SD. National EMS Agenda Writing Team. National EMS Research Agenda. Prehosp Emerg Care. 2002;6(3 Suppl):S1-43.

15. Maio RF, Garrison HG, Spaite DW, Desmond JS, Gregor MA, Stiell IG, et al. Emergency Medical Services Outcomes Project (EMSOP) IV: pain measurement in out-of-hospital outcomes research. Ann Emerg Med. 2002;40(2):172-9.

16. Joshipura M, Hyder AA, Rehmani R. Emergency care in South Asia: challenges and opportunities. J Coll Physicians Surg Pak. 2004;14(12):731-5.

17. Razzak JA, Kellermann AL. Emergency medical care in developing countries: is it worthwhile? Bull World Health Organ. 2002;80(11):900-5.

18. Ong ME, Shin SD, Tanaka H, Ma MH, Nishiuchi T, Lee EJ, et al. Rationale, methodology, and implementation of a dispatcher-assisted cardiopulmonary resuscitation trial in the Asia-Pacific (Pan-Asian Resuscitation Outcomes Study Phase 2). Prehosp Emerg Care. 2014;19(1):87-95.

19. Chan PS, McNally B, Tang F, Kellermann A. CARES Surveillance Group. Recent trends in survival from out-of-hospital cardiac arrest in the United States. Circulation. 2014;130(21):1876-82.

20. Ro YS, Shin SD, Song KJ, Park CB, Lee EJ, Ahn KO, et al. A comparison of outcomes of out-of-hospital cardiac arrest with non-cardiac etiology between emergency departments with low- and high-resuscitation case volume. Resuscitation. 2012;83(7):855-61.

21. Jönsson K, Fridlund B. A comparison of adherence to correctly documented triage level of critically ill patients between emergency department and the ambulance service nurses. Int Emerg Nurs. 2013;21(3):204-9.

22. Abrams HC, McNally B, Ong M, Moyer PH, Dyer KS. A composite model of survival from out-of-hospital cardiac arrest using the Cardiac Arrest Registry to Enhance Survival (CARES). Resuscitation. 2013;84(8):1093-8. 